\title{
Religião, identidade e prática política no reinado de Alfred de Wessex (871-899)
}

Monah Nascimento Pereira ${ }^{l}$

Resumo: A partir da análise da versão anglo-saxônica da $D e$ Consolatione Philosophiae, produzida entre os séculos IX e X no reino de Wessex e atribuída ao rei Alfred, procuraremos compreender o modo como se deu a criação de um ideal de identidade calcado na religião e na tradição latino cristã. Este ideal, em última instância, fortalece um novo modelo de prática real, apontando para um processo de unificação do reino, defesa ante aos invasores Vikings e integração de outras populações de acordo com interesses e necessidades reais. Observaremos a forma como a virtude cristã da sabedoria é recorrentemente retomada no texto, de modo a configurar uma busca por legitimação política a partir de um discurso de poder calcado na religiosidade cristã e seus preceitos.

Palavras-chave: Rei Alfred; identidade; Anglo-saxões.

Abstract: From the analysis of Anglo-Saxon version of $D e$ Consolatione Philosophiae, produced between the ninth and tenth centuries in the kingdom of Wessex and attributed to King Alfred, our aim will be to understand the creation of an ideal of identity based on religion and Latin Christian tradition. This ideal, ultimately strengthens a new model of royal practice, pointing to a process of unification of the kingdom, defence against the Viking invaders and integration of other populations according to royal interests and needs. We will research

\footnotetext{
${ }^{1}$ Mestranda em História na Universidade Federal do Paraná (UFPR). Bolsista CNPq.
} 
how the Christian virtue of wisdom is repeatedly discussed in the text, configuring a search for political legitimacy through a discourse of power based in the Christian religion and its precepts.

Key words: King Alfred, identity; Anglo-Saxons.

Tradicionalmente, ao se estudar o reinado de Alfred entre os anglo-saxões no século IX, remonta-se à Carlos Magno e o renascimento carolíngio do século anterior, de forma que pesquisadores buscam demonstrar as conexões entre tais contextos históricos. De fato, a aproximação entre ambos é evidente, tanto no que se refere a relações diplomáticas estabelecidas entre os francos e os reinos insulares de Mércia e de Wessex ${ }^{2}$, quanto na própria similaridade em alguns dos pontos centrais dos chamados "projetos educacionais" que teriam sido gestados por Carlos Magno e Alfred respectivamente. Entretanto, uma abordagem mais abrangente se faz possível, na medida em que observamos uma recorrente readaptação de valores e ideais romanogermânicos nos escritos tidos como alfredianos.

Notadamente, observa-se um esforço existente em tal contexto para que se traduzissem para a língua vernácula obras da Patrística Cristã. Estas foram produzidas originalmente, em grande medida, em

\footnotetext{
${ }^{2}$ A este respeito vide a carta enviada por Carlos Magno a Offa, rei dos Mércios, por exemplo. Apud.CROSSKEY-HOLLAND, Kevin (trad.). The Anglo-Saxon World-An Anthology. Oxford: Oxford University Press, 1999. pp. 189-191. Outro ponto a ser destacado é o próprio casamento do pai de Alfred com Judith, filha de Carlos, o Calvo, o qual evidencia as aproximações políticas e diplomáticas entre os reinos.
} 
um momento de construção e consolidação das monarquias romanobárbaras, as quais se pautavam em elementos clássicos, bem como em valores ditos "germânicos". Segundo Marcelo Cândido,

a grande contribuição da noção de Antiguidade Tardia é ter situado a instalação dos bárbaros no Ocidente menos em termos de fim do mundo romano do que de um rearranjo de forças que conduziu à constituição de um mundo ainda marcado pela influência da românia, mas profundamente original. $^{3}$

Nesse sentido, o reinado de Alfred bebe dessa mesma fonte, na medida em que retoma como voz de autoridade autores como Boécio e Orósio ${ }^{4}$. Na versão Alfrediana da Consolation of Philosophy, por

${ }^{3}$ SILVA, Marcelo Cândido. Entre "Antiguidade Tardia" e "Alta Idade Média”. In: Diálogos, DHI/PPH/UEM, v. 12, n. 2/n.3, 2008. p. 57.

${ }^{4}$ No que se refere a questão da autoria e datação das obras alfredianas a maioria dos estudiosos concorda que estes vários dos textos como Regula Pastoralis de Gregório Magno, De Consolatione Philosophiae de Boécio, Soliloquia de Santo Agostinho, teriam sido de autoria de uma mesma pessoa, ao que tudo indica Alfred, com o auxilio de um ou mais ajudantes (sendo Asser, seu cronista, um dos possíveis colaboradores. Entretanto há aqueles, como Godden, que questionam a autoria da De Cosolatione e Soliloquia, mas o autor não apresenta outra alternativa concreta para problema da autoria. De todo modo, como aponta Pratt, nesses trabalhos pode-se observar a presença de uma voz dominante, consistente em termos de estilo e coerentemente incluída no plano educacional e de traduções de Alfred, o qual inclui também artefatos de cultura material como a joia de Alfred. De toda forma, a própria atribuição de tais obras a Alfred ainda em tempos medievais, já no século $\mathrm{X}$, demonstra a conexão que se via ou que se queria fazer ver entre o rei e as traduções. Para mais informações sobre o assunto vide: GODDEN, Malcolm. Did King Alfred Write Anything? In: Medium AEvum 76.1, 2007. p 1-23; PRATT, David. Persuasion and Invention at the 
exemplo, percebemos como forte eixo temático a busca por sabedoria como guia da conduta do homem justo. Tal virtude é inserida no escopo dos preceitos cristãos, mas também remontando às noções clássicas segundo as quais Filosofia, uma das interlocutoras do texto original, e Sabedoria são a própria imagem da perfeição e, nesse caso, perfeição divina.

O prefácio da obra supracitada aponta logo de início que a tradução do latim para o inglês antigo foi feita de modo que o texto foi traduzido por vezes palavra por palavra, mas em outros momentos sentido por sentido (sense for sense), de forma a explicar o texto "on account of the various and multiple worldly cares which often busied him [Alfred] in mind and body"5. Destarte, percebe-se a busca por uma readaptação da obra de forma a melhor corresponder à sua realidade, de modo a re-significar valores e atributos provenientes de uma tradição clássico-cristã. Os assuntos práticos, mundanos, são apontados como relevantes para tradução da obra, que pode nos revelar pistas de uma

Court of King Alfred the Great. In: Catherine Cubitt (Ed.). Court Culture in the Early middle Ages: The Procedings of the First alcuin Conference. Turnhout: Brepols, 2003. p. 189-221. e DISCENZA, Nicole Guenther. The King's English: strategies of translation in the Old English Boethius. Albany: State University of New York, 2005. p. 139-140.

5 "Levando em conta as várias e múltiplas preocupações mundanas que muitas vezes ocuparam-no [Alfred] em mente e corpo". Livre Tradução. GODDEN, Malcolm e IRVINE, Susan (ed.). The Old English Boethius: An Edition of the Old English Versions of Boethius's De Consolatione Philosophiae (2 volumes). Oxford: Oxford University Press, 2009. p. 1. 
prática política específica na Inglaterra anglo-saxônica a partir de Alfred.

Ao se pensar política na era alfrediana, é preciso primeiramente compreender os mecanismos e as práticas exercidas no interior da sociedade política em questão. Nesse sentido, percebe-se que a prática política per se não pode ser deslocada de uma discussão teológica e filosófica. A teoria política observada no período em questão se pauta, em grande medida, em argumentos teológicos, cuja fonte maior é o cristianismo. Este é caracterizado por sua doutrina, moral e ritos, constituindo-se como uma filosofia revelada, que não se apresenta apenas em forma de discurso, mas que também leva a práticas concretas.

No caso de Alfred, compreendemos as diversas versões de clássicos da patrística produzidas ou atribuídas a seu reinado como uma busca por normatização de uma identidade, a qual é apresentada à sua sociedade política por meio de uma chamada tradução inventiva ${ }^{6}$ de tais obras para o inglês antigo. Várias delas eram de cunho filosófico, como a Consolation of Philosophy, de Boécio, a qual discute questões espirituais principalmente pelo foco da Sabedoria, grande interlocutora do diálogo apresentado no texto. Destarte, apresenta-se uma vinculação

\footnotetext{
${ }^{6}$ Segundo David Pratt, tais obras caracterizavam uma centralização real dos novos discursos de poder, os quais se pautavam em textos cuja autoria se encontrava monopolizada por atos de tradução inventiva. PRATT, David. The political thought of king Alfred the Great. Cambridge: Cambridge University Press, 2006. P. 339.
} 
à nível de tradição cultural e espiritual entre Alfred e essa reconhecida voz de autoridade, de maneira que a política se encontra intermeada na obra filosófica, na qual também são realçados elementos cristãos.

Tece-se assim um discurso de legitimação do rei, o qual se utiliza da atualização de valores clássicos e cristãos, objetivando a apresentação de um novo ideal identitário, pautado na valorização da instrução e da religião. Alfred não fora destinado a governar inicialmente, uma vez que era apenas o quinto filho varão e assumiu o trono somente após a morte de seus quatro irmãos mais velhos. Observa-se assim a necessidade ainda mais premente de se fortalecer a posição do mesmo como governante, o que foi feito através dos escritos produzidos na época.

De acordo com a visão isidoriana, a qual também é apresentada na Consolation, os escritos detêm uma grande importância no sentido de relatar o que ocorreu e são, portanto, verdadeiros. Na obra supracitada apresenta-se uma visão do papel daquele que escreve, criticando inclusive os maus escritores, os quais deixavam de cumprir sua função moral a contento.

[...] it has often very grievously happened through the misfortunes with writers that, because of their sloth and carelessness and lack of attention, they left unwritten the customs of those men and the deeds of 
those who in their day were most famous and most worthy of honour. ${ }^{7}$

O papel da escrita é assim visto como crucial para o registro daquilo que se passa e, portanto, esta pode ser considerada uma importante ferramenta de legitimação, fazendo circular discursos tecidos e aprovados por um rei com uma notável necessidade de sublimação de um certo defeito de nascimento, por assim dizer. Este, por vias sucessórias que valorizavam a primogenitura, não seria o rei mais indicado para ascender ao poder. Entretanto o uso da palavra escrita foi utilizado recorrentemente por Alfred para fortalecer sua posição como governante. Isso não foi feito apenas nos trabalhos de tradução a ele atribuídos, mas também e especialmente em outros textos, como a sua biografia régia, escrita pelo bispo galês Asser em aproximadamente 893. Nesta, percebemos também a constante associação de Alfred com as virtudes religiosas e com a própria religião em si, tendo sido ele apontado na obra, ainda quando pequeno em visita a Roma, como o favorito do próprio Papa.

In the same year [853] King Æthelwulf sent his son Alfred to Rome in state, accompanied by a great

\footnotetext{
7 "Com frequência ocorreu muito gravemente através dos infortúnios com escritores que, por causa de sua preguiça e descuido e falta de atenção, deixaram de escrever os costumes e feitos daqueles que em sua época, foram os mais famosos e mais dignos de honra." (Livre Tradução) GODDEN, Malcolm e IRVINE, Susan (ed.). Op. Cit. P. 28.
} 
number of both nobles and commoners. At this time the lord Pope Leo was ruling the apostolic see; he anointed the child Alfred as king, ordaining him properly, received him as an adoptive son and confirmed him. ${ }^{8}$

Tal menção demonstra uma tentativa de utilização da voz de autoridade do Papa para confirmar ou legitimar o reinado de Alfred, o qual apresentava uma forte identificação com o catolicismo, ou seja, a prática reta do cristianismo. Em uma carta enviada ao pai de Alfred, o papa Leão apresenta sua visão a respeito do encontro com o menino, a qual difere um pouco daquela mencionada na biografia.

We have now graciously received your son Alfred, whom you were so anxious to send at this time to the threshold of the Holy Apostles, and we have decorated him, as a spiritual son, with the dignity of the belt [or sword] and the vestments of the consulate, as is customary with Roman consuls, because he gave himself into our hands. ${ }^{9}$

8 "No mesmo ano [853] o Rei Æthelwulf enviou seu filho Alfred a Roma, acompanhado por um grande número de ambos os nobres e pessoas comuns. Neste momento, o senhor papa Leão estava governando a Sé Apostólica; ele ungiu a criança Alfred como rei, ordenando-o corretamente, recebeu-o como um filho adotivo e confirmou-o." (Livre Tradução) KEYNES, Simon e LAPIDGE, Michael. Alfred the Great-Asser's life of king Alfred and other contemporary sources. London: Penguin, 1983. P. 69.

9 "Recebemos graciosamente seu filho Alfred, a quem você estava tão ansioso para enviar neste momento para a soleira dos Santos Apóstolos, e o decoramos, como um filho espiritual, com a dignidade do cinto [ou a espada] e os paramentos do consulado, como é costume com cônsules romanos, porque ele deu-se a si mesmo em nossas 
Assim sendo, Keynes e Lapidge salientam que "the chronicler probably intended merely to convey that Alfred had been marked out for kingship when still a young boy, in much the same way as heavenly signs attended the birth of those who were to become saints" ${ }^{10}$. Não se trataria apenas de uma legitimação por sangue assim, questão essa que inclusive é relativizada na Consolation, por exemplo, mas também por predestinação. De acordo com a concepção teleológica e providencialista de história presente nos escritos alfredianos, Alfred seria o melhor rei e demonstraria isso por suas práticas de governo, eficazes tanto no campo das armas como no campo das letras e da fé.

Nesse sentido elencamos como fonte primária para nossa análise a tradução para o inglês antigo da De Consolatione Philosophiae, originalmente escrita em latim por volta do ano de 524, três séculos antes. A Consolatione é um tratado filosófico escrito por Boécio enquanto este se encontrava, ao que tudo indica, aprisionado por traição ao então imperador godo Teodorico. No texto, Boécio se encontra desiludido com sua fortuna adversa, ao que é confrontado pela Filosofia, que busca confortá-lo e demonstrar que apesar dos infortúnios

mãos.” (Livre Tradução) POPE LEO IV, Apud. KEYNES, Simon e LAPIDGE Michael. Op. Cit. p. 232.

10 "O cronista, provavelmente, tinha apenas por objetivo transmitir que Alfred havia sido marcado para a realeza quando ainda garoto, da mesma forma que os sinais celestiais marcavam o nascimento dos que viriam a se tornar santos." (Livre Tradução) KEYNES, Simon e LAPIDGE, Michael. Op. Cit. p. 232. 
vividos pelo autor este sempre estaria amparado por seu conhecimento e sua razão.

O sentido atribuído à obra original em sua versão anglosaxônica aponta que o objetivo de Boécio, ao se colocar contra Teodorico, era o de recuperar os antigos direitos romanos e também a própria Cristandade, exemplificando justamente dois dos grandes pilares dos textos traduzidos no período: uma tradição clássica e os preceitos cristãos. Considerando-se a associação da Consolation com o reinado de Alfred, nota-se assim que tais pilares também servem ao objetivo de consolidar ante a sociedade política uma visão específica acerca de uma cultura política, na qual a legitimidade se dá pela virtude mais do que apenas pela genealogia. Assim, quando Teodorico é mencionado, não se ignora a questão da ancestralidade, mas esta não tem primazia sobre suas práticas religiosas; "this Theodoric was of the line of Amul. He was Christian, although he persisted in the Arian heresy"11. A tradicional identidade de linhagem, estirpe, é assim reconhecida, mas passa a ser relativizada pela heresia ariana, de modo a valorizar a religião como base principal da criação de uma identidade anglo-saxônica.

\footnotetext{
11 "Este Teodorico era da linhagem dos Amal. Era cristão, embora persistisse na heresia ariana." (Livre Tradução) GODDEN, Malcolm e IRVINE, Susan (ed.). Op Cit. p. 4.
} 
No quinto capítulo da obra, Sabedoria e Razão se dirigem a Boécio, afirmando:

I realised that you had departed from your father's country, that is, from my teachings. [...] Noone should have expected such thing of you either, if you were willing to recall of what birth you were and of what citizenry, as regards the world, or again, in a spiritual sense, of what community you were in your mind and in your reason, that is that you are one of the righteous and well-intentioned (those are the citizens of the heavenly Jerusalem) ${ }^{12}$.

Nessa passagem, há a criação de uma identidade comum para os homens bons, ou no sentido salomônico proveniente da sabedoria, de homem sábio. $\mathrm{O}$ nascimento é levado em consideração, mas o verdadeiro pai de Boécio seria a própria sabedoria em si, por meio da qual ele teria conseguido toda sua riqueza e dignidade ${ }^{13}$. Em última instância, a forma pela qual o indivíduo consegue atingir a sabedoria é através do treinamento e instrução. Ora, a tradução de obras tradicionais latinas para a língua vernácula, que começa a ocorrer de forma mais

\footnotetext{
12 "Percebi que você tinha se afastado dos domínio de seu pai, ou seja, de meus ensinamentos. [...] Ninguém tampouco teria esperado tal coisa de você, se você estivesse disposto a se lembrar de que nascimento você era e de que cidadania, no que diz respeito ao mundo, ou mais uma vez, em um sentido espiritual, do que comunidade você era em sua mente e em sua razão, ou seja que você é um dos justos e bem-intencionado (esses são os cidadãos da Jerusalém celeste)" (Livre Tradução) GODDEN, Malcolm e IRVINE, Susan (ed.). Op Cit. p. 7. ${ }^{13}$ Idem. p. 11.
} 
marcante a partir de Alfred, visava justamente difundir entre os ealdormen e thegns ${ }^{14}$ os conhecimentos autorizados considerados de extrema importância, isto é, virtudes cristãs tais quais sabedoria, a mãe de todas as virtudes, além de cautela, moderação, coragem e justiça ${ }^{15}$. Caracteriza-se assim um discurso de poder que visa apresentar moldes de conduta e pensamento para uma sociedade política que tradicionalmente, durante o medievo, precisa ser conquistada pelos governantes, uma vez que sem seu apoio o exercício do poder se torna extremamente difícil.

Sabedoria e Razão exprimem então seu desejo de colocar a Mente (personagem que substitui o original Boécio durante boa parte do texto) no bom caminho, o caminho do saber; "so I would wish, Mind,

\footnotetext{
${ }^{14}$ Esta foi uma terminologia que surgiu como a versão vernácula equivalente a vários títulos em latim, como princeps, dux, comes, praefectus. Ou seja, tratava-se de uma nomenclatura genérica usada para designar homens de grande importância que detinham autoridade, muitas vezes referindo-se a títulos quase reais. Entretanto, o significado da palavra foi se alterando ao longo do tempo e, no período alfrediano, ealdorman era utilizado para definir líderes de condados individuais. Dessa forma, ealdorman era, assim como o termo thegn, utilizado amplamente tanto como caracterização de uma nobreza quanto de personagens de autoridade subordinados ao rei, de forma que o status dessas pessoas era principalmente bem definido no que se refere a uma inferioridade com relação a Alfred, por exemplo, que detinha o título real.

${ }^{15}$ A esse respeito, vide From the translation of Boethius's Consolation of Philosophy. Apud. KEYNES, Simon e LAPIDGE, Michael. Alfred the Great-Asser's life of king Alfred and other contemporary sources. London: Penguin, 1983. p. 133. Essa é uma das passagens acrescentadas na versão anglo-saxônica, não presente no texto original em latim, o que demonstra uma preocupação específica da época em enumerar as virtudes segundo as quais os homens bons deveriam se comportar, todas elas em coordenância com os preceitos cristãos.
} 
that you came up to us if you wished, on condition that you afterwards with us are willing to seek the earth for the sake of good people"16. A Sabedoria então convida Boécio a juntar-se a ela novamente, mas sempre tendo em mente o bem das boas pessoas, como que em uma missão, da qual o homem sábio é o instrumento, tal qual o ideal de rei sábio presente na tradição salomônica, também incorporada desde o contexto carolíngio.

Tal obra de cunho filosófico apresenta assim um acentuado tom cristão, pelo qual se procura definir uma identidade aos anglosaxões, que vai além das questões de ancestralidade. Essa forma de pensar justifica a legitimidade de um rei voltado para as questões da alma e coloca a religião como base de um ideal identitário e cultural que se quer transmitir ao reino. Nesse sentido, em última instância, não são apenas laços familiares, sanguíneos ou étnicos que definem um homem e sim as virtudes que este possui. Na Consolation a Sabedoria cita o sogro de Boécio, Symmachus, e lembra-o de quão caro este era para o aprisionado, mas isso não por laços familiares, necessariamente, mas por suas qualidades a citar: sabedoria e demais virtudes. Da mesma maneira sua filha, esposa de Boécio, seria uma mulher cheia de

\footnotetext{
16 "Então eu gostaria, Mente, que você viesse até nós, se assim desejasse, desde que você posteriormente conosco esteja disposto a buscar a terra para o benefício das boas pessoas" (Livre Tradução) GODDEN, Malcolm e IRVINE, Susan (ed.). Op Cit. p. 12.
} 
prudência, modéstia e pureza, e os filhos do casal também possuíam as virtudes do pai e do avô, "as like old man as young can be"17.

Sabedoria se refere aos Romanos como os ancestrais dos homens e estes não eram intrinsecamente bons por sua estirpe, uma vez que as ações e a conduta podem fazer com que o homem se desvie do bom caminho; "your ${ }^{18}$ ancestors of old, the counsellors of the Romans, first removed the kingly name from Rome in the days of Tarquin, the proud king, because of his arrogance" ${ }^{\prime 1}$. Assim sendo, o poder é tão bom quanto aquele que o exerce, ou segundo o texto "power is never good unless he who has it is good" 20 . A autoridade advém da virtude e da excelência e não o contrário, pois o que define um homem é sua prática reta.

É claro que este tratava-se de um ideal difundido especialmente para uma elite e não era necessariamente uma prática presente entre a população como um todo. A ideia de uma identidade forjada nas bases do cristianismo e a própria organização social decorrente disso eram instrumentais para o governo de Alfred e sua

17 "Tão parecidos com os homens velhos quanto os jovens podem ser." (Livre Tradução) Idem. p. 15.

${ }^{18}$ Este "your" em itálico refere-se aos homens como um todo, no plural, e não apenas Boécio mas aqueles de seu meio também.

19 "seus antepassados de tempos antigos, os conselheiros dos romanos, primeiro removeram o nome real de Roma nos dias de Tarquino, o rei orgulhoso, por causa de sua arrogância." (Livre Tradução) GODDEN, Malcolm e IRVINE, Susan (ed.). Op Cit. p. 23.

20 "O poder nunca é bom, a não ser que aquele que o possui seja bom." (Livre Tradução) Idem. p. 23. 
descendência, os quais buscavam legitimação de sua linhagem, uma constante na Idade Média. Além disso, observa-se neste contexto uma soma de fatores que ajudaram a moldar um novo cenário político na ilha, de modo que Alfred teve de enfrentar dificuldades ante aos inimigos Vikings e também acabou por buscar estender sua hegemonia para além de Wessex, de modo a não ser mais considerado apenas rei dos saxões do oeste, mas sim rei dos anglo-saxões ${ }^{21}$.

No que se refere a um projeto de organização social pautado no cristianismo, é notável a presença de uma alusão à sociedade tripartida, mais de um século antes do que preceituou Adalberon de Laon a respeito do tema. Discorre a Mente:

Truly you know that no-one can show any skill, or exercise or control any power, without tools and material. The material of any skill is that without which one cannot exercise that skill. Then the material for a king and his tools for ruling with are that he has his land fully manned. He must have prayer-men and army-men and work-men ${ }^{22}$.

${ }^{21}$ A esse respeito, vide PEREIRA, Monah N. Warfare and Wisdom - as bases legitimadoras da monarquia Anglo-Saxã sob Alfredo, o Grande (871-899). Monografia de conclusão de curso. 2010.

22 "Verdadeiramente você sabe que ninguém pode demostrar qualquer habilidade, ou exercitar ou controlar qualquer poder, sem ferramentas e material. $\mathrm{O}$ material de qualquer habilidade é aquele, sem o qual não se pode exercer essa habilidade. Então o material para um rei e os seus instrumentos para governar são de que ele tenha a sua terra totalmente tripulada. Ele deve ter homens de oração e homens de armas e 
Tal passagem demonstra uma característica medieval que vem se desenvolvendo mais fortemente a partir do período alfrediano, segundo a qual a Igreja passa a ser um denominador comum para as interações socioculturais. A explicação religiosa para uma sociedade tripartida denota a importância da religião para a definição identitária de um povo, uma vez que os anglo-saxões, sob o reinado de Alfred, devem se comportar de tal forma a disponibilizar para seu bom e sábio rei as ferramentas necessárias para bem governar. Novamente percebemos um projeto de poder e de prática real que se entrelaça com a produção cultural do período.

Nesse sentido, a ancestralidade e linhagem são relativizadas, de forma que conceitos classicamente valorizados pela etnogenese ${ }^{23}$ não seriam o fator único e preponderante na definição do caráter de um homem da identidade de um grupo. Esta se deveria, de acordo com o ideal alfrediano, a uma unidade cristã atingida através da formação, muito na linha da paideia e humanitas.

He who wants to have complete power must first strive to have power over his own mind [...]

homens de trabalho" (Livre Tradução) GODDEN, Malcolm e IRVINE, Susan (ed.). Op Cit. p. 26.

${ }^{23}$ A esse respeito, vide os trabalhos de Andrew Gillet, "On Barbarian Identity" e Herwig Wolfram, "Origo et Religio”, na coletânea de Thomas Noble, "From Roman provinces to Medieval kingdoms". 
Therefore I urge you that you rejoice over other men's goodness and their noble birth to the extent that you do not claim it for your own, for every man's goodness and his nobility is more in the mind than in the flesh ${ }^{24}$.

A obra aponta que todos os homens vieram de um pai e uma mãe, e que, em última instância, Deus é o supremo pai de todos os homens $^{25}$, por isso a nobreza se dá pela educação da mente e busca pela sabedoria mais do que por parentesco. A preocupação em colocar a religião como a baliza para identificação dos homens nobres é bastante importante em um contexto no qual observam-se intensas interações culturais entre escandinavos e anglo-saxões, sendo que muitos dos nórdicos já haviam abandonado suas atividades predatórias Vikings em favor de assentamento na ilha. $\mathrm{O}$ volume de elementos até então considerados externos e a crescente interação entre estes e a população local levam a delimitar barreiras étnicas, mas também criar princípios de regulação para interação entre os diferentes povos que passam a dividir o mesmo espaço.

\footnotetext{
24 “Aquele que quer ter poder total deve primeiro se esforçar para ter poder sobre sua própria mente [...] Por isso peço-lhe que se alegre com a bondade de outros homens e sua origem nobre, na medida em que não reclame-a para si próprio, pois a bondade de cada homem e sua nobreza está mais na mente do que na carne" (Livre Tradução) GODDEN, Malcolm e IRVINE, Susan (ed.). Op Cit. pp. 44-45. ${ }^{25}$ Idem. P. 45.
} 
Ao apontar a fé cristã como baluarte da sua identidade e legitimação, tal fé não inclui a heresia ariana, uma vez que é dito explicitamente que "God is single and undivided"26. De acordo com este raciocínio, pagãos e arianos estariam excluídos. Nesse sentido, a sabedoria cristã reta seria a única forma de se chegar à uma unidade, característica do divino e aspiração do reino. Aqueles que buscam a recompensa em Deus e não na fama terrena podem de fato aproximar-se dessa universalidade; os homens seriam muito diferentes uns dos outros e muito passageiros, mas a sabedoria vem de Deus. Podemos observar a importância dada a tais questões religiosas em relação a questões étnicas quando observamos o episódio do fim da guerra entre Alfred e o rei nórdico Guthrum, o qual dominava a Ânglia Oriental na década de 870.

Na Anglo-Saxon Chronicle, descreve-se a cristianização de Guthrum em 878 após este ter sido derrotado pelo exército de Alfred, processo em que o próprio rei anglo-saxão teria sido o padrinho de batismo do até então inimigo: "Three weeks later King Guthrum with 30 of the men who were the most important in the army came [to him] at Aller [...] and the king stood sponsor to him at his baptism there; and the unbinding of the chrism took place at Wedmore"27. Ainda mais

\footnotetext{
26 "Deus é único e indivisível" (Livre Tradução) GODDEN, Malcolm e IRVINE, Susan (ed.). Op Cit. p. 48.

27 "Três semanas mais tarde o rei Guthrum com 30 dos homens que eram os mais importantes no exército veio [até ele] em Aller [...] e o rei tornou-se padrinho no seu 
interessante que a prática relativamente comum de conversão de reis derrotados é o tratado firmado entre ambos os reis, no qual se diz que todos os anglo-saxões bem como todas as pessoas instaladas em Ânglia Oriental haviam concordado com os termos. Apesar da distinção aqui existente entre os de "raça inglesa" 28 e os demais, os termos do tratado fixam um valor igual de compensação pela morte de qualquer destes homens; "if a man is slain, all of us estimate Englishman and Dane at the same amount, at eight half-marks of pure gold",29.

Assim sendo, Alfred estende sua hegemonia sobre essas pessoas, aceitando-as sob sua tutela mediante sua conversão ao cristianismo, grande pilar identitário de seu governo. Tal prática justificaria a hegemonia e a unidade apenas alcançáveis através de Deus, ou seja, do cristianismo.

\section{Bibliografia:}

ABELS, Richard P. Alfred the Great: War, Kingship and Culture in AngloSaxon England. Harlow: Longman, 1998.

batismo lá; laço da crisma teve lugar em Wedmore." (Livre Tradução) The AngloSaxon Chronicle. Apud. CROSSKEY-HOLLAND, Kevin (trad.). Op. Cit. p. 41.

${ }^{28}$ Apud. KEYNES, Simon e LAPIDGE, Michael. Alfred the Great-Asser's life of king Alfred and other contemporary sources. London: Penguin, 1983. p. 171.

29 'Se um homem é morto, todos nós estimamos a mesma quantia para 'Ingleses' e 'Dinamarqueses', a qual será de oito half-marks de ouro puro" (Livre Tradução) Idem. P. 171. 
BLAIR, John. The Church in Anglo-Saxon society. Oxford: Oxford University Press, 2005.

BLAIR, Peter Hunter. An introduction to Anglo-Saxon England. Cambridge: Cambridge University Press, 2003.

CARNICELLI, Thomas. King Alfred's Version of St. Augustine's Soliloquies. CROSSKEY-HOLLAND, Kevin (trad.). The Anglo-Saxon World - An Anthology. Oxford: Oxford University Press, 1999.

DISCENZA, Nicole Guenther. The King's English: strategies of translation in the Old English Boethius. Albany: State University of New York, 2005.

GODDEN, Malcolm. Did King Alfred Write Anything? In: Medium Avum 76.1, 2007. p 1-23. e IRVINE, Susan (ed.). The Old English Boethius: An Edition of the Old English Versions of Boethius's De Consolatione Philosophiae (2 volumes). Oxford: Oxford University Press, 2009.

GREENFIELD, Stanley B. e CALDER, Daniel G. A new critical history of Old English literature. New York: New York University Press, 1986.

JAMES, Edward. Europe's Barabrians. Routledge, 2009.

KEYNES, Simon e LAPIDGE, Michael. Alfred the Great-Asser's life of king Alfred and other contemporary sources. London: Penguin, 1983.

LAPIDGE, Michael (ed.). The Blackwell Encyclopedia of Anglo-Saxon England. Blackwell Publishing, 2000.

MEDEIROS, Elton O. S. Alfredo o Grande e a linhagem sagrada de Wessex: a construção de um mito de origem na Inglaterra anglo-saxônica. In: COSTA, Ricardo da (coord.). Mirabilia 13 - As relações entre História e Literatura no mundo Antigo e Medieval. 2011. p. 134-172. 
NOBLE, Thomas (ed.). From Roman provinces to Medieval Kingdoms. Routledge, 2006.

PEREIRA, Monah N. Warfare and Wisdom - as bases legitimadoras da monarquia Anglo-Saxã sob Alfredo, o Grande (871-899). Monografia de conclusão de curso. 2010.

PRATT, David. The political thought of king Alfred the Great. Cambridge: Cambridge University Press, 2006.

REUTER, Timothy (Ed.). Alfred the Great - Papers from the EleventhCentenary Conferences. Ashgate, 2003.

SEDGEFIELD, Walter John (trad.). King Alfred's Version of the Consolations of Boethius. Kessinger Publishing's Legacy Reprints.

SILVA, Marcelo Cândido. Entre "Antiguidade Tardia" e "Alta Idade Média”. In: Diálogos, DHI/PPH/UEM, v. 12, n. 2/n.3, 2008. 